9 cases were notified. Assuming the preventive measures were efficacious, it is interesting to consider which was most likely to be efficiently performed.

Flies still swarmed after the epidemic ceased. The spraying of " contacts," although advocated, was not supervised and could not be insisted on. The probability is that this measure was neglected in many instances, and cannot be reckoned as a potent factor in staying the spread of infection. Isolation was observed as far as possible. But in many instances parents refused to send their children to the isolation hospital, only four patients being admitted. The only measure thoroughly and regularly performed was watering the streets with antiseptics, and in this way rendering the dust innocuous. The evidence from this epidemic, therefore, would appear to support the view that dust infection is an important factor in spreading the disease.

Clinical Symptoms.

The symptoms differed in no material respect from those already published in the British Medical Journal of November 4th, as typical of the epidemics in Cornwall, Huntingdon and Devonshire.

In the majority of cases gastro-intestinal trouble either preceded or accompanied the attack. In one instance amongst my own cases the illness was preceded by tonsillitis. Headache, pain at the back of the neck, furred tongue, vomiting, usually constipation, with some fever, marked the onset of the disease. In bad cases muscular twitching and convulsions occurred, also retraction of the head and spinal rigidity. Paralysis of one or more limbs supervened in from two to three days. Kernig's sign was present in 75 per cent. of the cases. Reflexes were usually abolished with the onset of paralysis. Retention of urine occurred in 10 per cent. of the cases. Pain and tenderness was usually present in the paralysed limbs. In some cases hyperaesthesia was very marked. The paralysis in the majority of instances appears to be permanent.

$$
\text { Bacteriology. }
$$

In several cases the spinal fluid was examined, and Dr. Heath, county medical officer of health, has obtained some interesting results, which will be published in due course. Dr. Alice Taylor also worked at the bacteriology of the lisease, and her results appeared to confirm those of Dr. Heath.

Treatment.

Various drugs were tried, including urotropine, but the course of the disease appeared to be uninfluenced. No doubt with the isolation of the specific organism, and preparation of a vaccine, better results will be obtained.

\section{Notes of Cases.}

H. D., aged 19. On Augast 10h suffered from tonsillitis, H. D., aged 19. On August 10th suffered from tonsillitis,
which passed off in a few days. On August 19th complained of which passed off in a few days. On August 19th complained of headache, especially at the back of the neck, vomiting, and
pains in limbs and back. Temperature $100^{\circ}$. Tongue furred ; pains in limbs and back. Temperature $100^{\circ}$. Tongue furred; leg. Kernig's sign present-paralysis of left arm severe. At the present time, three-and-a-half months from onset, has not recovered full use of a.rm, and is still slightly lame.

C. O., aged 5, commenced August 19th. Much pain in back of head and down the spine. Retraction of head and spinal rigidity very marked-some twitchings of muscles during sleep. Kernig's sign present; reflexes abolished. Hyperaesthesia of paralysed limbs. The case is interesting, as, although cerebrospinal symptoms were well marked, examination failed to confirm the diagnosis of cerebro-spinal feve

Mrs. B., aged 40, taken ill September 14th, and moved into

Mrs. B., aged 40, taken ill September 14th, and moved into
hospital. Paralysed in both legs-very complete; no hyperaeshospital. Paralysed in both legs-very complete; no hyperaesthesia. Kernig's sign absent; reflexes absent; temperature
from $100^{\circ}$ to $102^{\circ}$. Retention of urine for ten days. She died from $100^{\circ}$ to $102^{\circ}$, Retention of urine for ten days. She died Examination of spinal tluid was negative, as far as cerebrospinal fever was concerned. This patient was certain she contracted the disease from one of her children, whom she said was ill a week before she was, with vomiting and headache. The child was not attended by a doctor, and when seen later showed no paralysis, but this may have been an abortive case.

F. R., aged 4. Commenced with vomiting and headache, and pain in the back, on September 4th. Paralysis of both legs. Kernig's sign present; hyperaesthesia present; constipated. Reflexes absent. Paralysis very complete. At present time there is some slight return of power in the flexors. Exthere is some slight return of power in the flexors. Exfever.

S. C., aged 5. Seen September 14th. Left leg paralysed. Had had slight attack of headache, pain in back of neck, and vomiting three days previously. Doctor only called in when paralysis noticed. Uninterrupted recovery. Cerebro-spinal tluid negative.

\section{A RECORD OF \\ SOME CASES OF EPIDEMIC PARALYSIS OCCURRING IN HAMPSHJRE."}

By G. FULLER ENGLAND, M.D.Cantar.,

PHYSICIAN, ROYAL HAMPSHIRE COUNTY HOSPITAL, WINCHESTER.

I PRopose shortly to narrate 7 cases of acute disease affecting either the spinal cord or the meninges, or both, which $\mathbf{I}$ have personally seen between the beginning of September and the middle of November. I have purposely described these cases under the general heading of "Epidemic Paralysis," as in my opinion, although two, at least, may, perhaps, be considered to approximate the description of cases of acute anterior poliomyelitis, even these presented some unusual features. In view of the fact that the county medical officer has asked for a return of all such cases in his area, it is of more than ordinary interest to note that, in addition to the cases mentioned in this paper, I had two other undoubted instances of acute poliomyelitis admitted into hospital within the last six weeks, the date on which this note was made being October 16th, and I know of at least two or three others.

CASE I.

R. B. was taken ill on September 6 th, but Mr. Way was not sent for until September llth, when he found him complaining of great pain in the head and back of the neck, and aching pains and stiffness all over. Weakness of the legs was so great that he could hardly stand. The temperature was $101^{\circ}$. On September 12th the stiffness had increased and the pain concentrated in the back of the hearl and neck; it was very acute, and the head was very markedly retracted. The temperature was slightly lower. The breathing was hurried and jerky. The slightly lower. The breathing was hurried and jerky. The
knee-jerks were absent. Babinski's sign was absent, but knee-jerks were absent. Babinski's sign was absent, but Kernig's sign was marked on attempting to extend the knee on the flexed thigh. The stiffness and pain in the neck muscles rendered the patient unable to

On September 13th, when I saw him in consultation with Mr. Way, this condition obtained, but he was evidently much worse. He was perspiring freely all over, was of a dusky, cyanotic colour, and had a rapid and very soft pulse, but no cough; breathing was shallow and very irregular; there was acute congestion of both bases; the temperature was subnormal. There was considerable loss of power in the arms, but no sign of involvement of the facial nerves, and he was fully conscious and able to answer questions, though acutely ill. He died the same evening, apparently from paralysis of the phrenics.

His history was that he had been apparently well until His history was that he had been apparently well until
September 6 th, when, after a long bicycle ride in the great heat, he felt ill and feverish, and went to bed shivering and complainhe felt ill and feverish, and went to

It is interesting to note that there was a definite history of a similar but milder attack, in which much the same symptoms were complained of a month earlier, from which he completely recovered without medical aid.

$$
\text { CASE II. }
$$

The next case was a girl, D. P., aged 6 , who a few weeks previously had been under my care for an attack of the gastroenteritis which prevailed during the hot weather. On Sepenteritis which prevalled into the river, but her mother had given her a hot bath and wrapped her in warm blankets, and she was apparently none the worse for her adventure.

On or about the September 17th it was noticed that her walking was stiff and her gait somewhat unsteady, and on September 19th she complained of backache, and was apparently feverish and out of sorts. On September 20 th the feverish attack was distinct and her eyes were suffused and bloodshot. She complained of no pain unable to turn over in bed.

On September 2lst fever still continued and I was asked to see her, but was not told of any of the above symptoms or history, except that she had had a feverish cold. She was always a pale, fragile-looking child. There was nothing to indicate anything of a more serious nature, so I must confess to having been caught napping until I was sent for again on September 25 th, when I found her lying on her side with her legs drawn up and her head somewhat retracted, complaining of great tenderness all over, and especially of acute pain in the egs when touched, pain in the back, and inability to raise herself at all from the bed. Any attempt to straighten her limbs caused her to cry out loudly. She could not stand. The knee-jerks were absent. Her temperature was normal; she was not tlushed, nor was there any rash. She could not sit up,
and began to cry directly she was raised in the bed. There was some delay in performing the act of micturition.

She was removed to hospital, where after a few days her condition began to improve and she could straighten the legs without much pain. It was then found that the peroneal group of the left leg was paralysed. A note on October 16th group of the left leg was paralysed. A note on October 16th
says that "There is a good deal of wasting of both legs, but says that "There is a good deal of wasting of both legs, but
she can now move them quite freely and sit up in bed without * ${ }^{*}$ Read before the winchester Division of the British Medical Association. 
pain." As the acute febrile condition had subsided and she is a nervous child I abstained from lumbar puncture, though had she become worse I should have drawn off some spinal fluid for examination. The feature in her case is the extreme rigidity of the ham-strings and calf muscles of both legs and the general weakness of the back and shoulder muscles. The reflexes are still absent. Babinski's sign was never present.

The paralysed peronei are already (October 17th) recovering under massage and faradism. She has had no elevation of temperature since admission to the hospital.

\section{CASE III.}

On September 27th I was called to B. B., a girl aged 4, who had been taken ill on September 18th with pains in the head, neck, and limbs. She also complained of stomachache and was veck, and limbs. She also complained of stomachache and was the face and that she was unable to speak clearly. She was unable to cough or sneeze and her breathing was gasping in unable to cough or sneeze and her breathing was gasping in
character. She was quite unable to sit up and cried when any attempt was made to raise her.

When I saw her on September 27 th she was asleep, and I was at once struck by a curious catch in her breathing about every rather shallow, and her colour good.

On September 30th she complained of pains in the left arm and leg and especially in the back of the neck, and was unable to raise her head from the pillow. The knee-jerks were absent, but there was no wasting or paralysis of the legs. The plantar reflexes were present. Babinski's and Kernig's signs were absent; there was no eruption, and her temperature was not absent;
raised.

She slowly improved; breathing on October 15th was normal and the knee-jerks had returned, but there was a marked flaccidity of the muscles of the shoulder girdle, though no actual paralysis. On lifting her up under the armpits the shoulders seemed to slip upwards owing to weakness of the rhomboids and latissimus dorsi (the phenomenon so generally obtained in pseudo-hypertrophic paralysis) was very mark
but she could now walk well, though somewhat unsteadily.

During August she had had a so-called bilious attack, in which she complained of pain in the head and neck, and vomited. I was not at that time asked to see her.

\section{CASE IV.}

B. O., a boy aged 5, I saw in consultation with Dr. Whittindale of Bishop's Waltham, who has kindly sent me the dale of Bishop's

"On September 17th he was taken with sudden acute pain in the head and back of the neck, followed by general malaise and pains in the arms and legs. His mother says the child was Tretful and feverish for some days. First seen September 22nd. tion of head. Slight opisthotonos; acute pain in neck, back, and legs. Legs drawn up. Kernig's sign marked. Tache cérébrale present. No hyperaesthesia. Constipation. Patellar reflexes absent. September 24th. Rapid catching breathing; general râles all over chest. September 27th. Marked head faeces passed involuntarily."

When I saw the patient on September 29th I had nothing to add to Dr. Whittindale's careful notes. The boy was extremely ill, and the condition was just as he has described for Sepill, and the condition was just as he has described for Septo clear up and that he had recovered the use of bladder and rectum, was fully conscious and able to answer questions, I ventured to give a hopefnl prognosis, but stated that in my opinion it would be some weeks before he would be really convalescent.

On October 13th Dr. Whittindale reports that " the boy is slowly improving. The attacks of pain come on three or four times daily only; he can almost put his feet down straight, but Kernig's sign is still marked, and he cannot get his chin to the sternum; breathing normal, deep reflexes absent. General condition much improved.

He was treated with bromides, and at my suggestion a small dose of strychnine and 3 grains of urotropin were added to the mixture to be taken every six hours; strychnine was indicated mixture to be taken every six hours

CASE v.

A. G., 3 years old in November, 1911, seemed a little tired on the afternoon of October 12th. She had an attack of sudden pain at 5 a.m. on October 13th, crying, putting her hands to the back of the neck, exclaiming, "Can't move my head, Nanny." The head was retracted. She lay on her back with the knees drawn up. Kernig's sign was not present, but there was sligh rigidity. Breathing was very shallow, jerky, and catchy, bu there was no alteration in colour. The temperature was subnormal, and the pulse 120 . There had been no vomiting. Poultices were applied at once, and 6 grains of urotropin in milk given at 8 o'clock. The urotropin was continued ever four hours in doses of 3 grains. There were involuntary spasm speech or difficulty in swallowing. Jerky movements of both arms were present, but there was no tache cérébrale. Profuse arms were present, but there was no tach
sweating was possibly due to poultices.

The knee jerk was obtained with difficulty on the left side; it was ausent on the right. Babinski's sign was not present. 'There was some vomiting during October 14th, but on the folw'parently quite well. On October 16th there were slight twitchings of the arms, and she seemed otherwlse very well; a rather prolonged attack of twitchings occurred in the evening, the patient being somewhat constipated at the time. After a
good sleep and satisfactory action of the bowels these symptoms good sleep and satis

I will supplement this series by 3 cases now in hospital -2 under my colleague, Dr. Tullis; the third sent to me by Dr. Hodgson of Alresford.

\section{Hospital CaSe I.}

T. T., aged 14, towards the end of August was sitting in a chair one evening at the end of his day's work, when he was suddenly taken with acute pain in the head, neck, and back, and was unable to rise from his chair. He had felt quite and was unable to rise from his chair. He had felt quite well all day. He was unable to sleep that night owing to the
acuteness of the pains in the spine and legs, and the followin acuteness of the pains in the spine and legs, and the following day was unable to move either arms or legs. The pains lasted
four or five days. He regained a certain amount of power in four or five days. He regained a certain amount of power in
about two days' time, but was unable to lift the left arm above the head, though he could raise the forearm to the mouth.

When admitted on October 6th the triceps and muscles of the left shoulder girdle were the parts chiefly affected. Of the lower limbs the left leg was the worse, the thigh muscles being chiefly involved; he was unable to draw up the left leg. The knee-jerks were absent on both sides. He could not bear any attempt at extensio

The temperature ranged from subnormal to 99.6. ${ }^{\circ}$ A lumbar punctnre was done shortly after admission, the report from the Clinical Research Association being as follows:

"The fluid is faintly alkaline but fairly albuminous ; it shows a normal reduction with Fehling's solution. The centrifugalized deposit is small in amount, and consists of a few blood discs, an extremely small number of leucocytes, chiefly of the lymphocyte type, and a little, if any, in excess of the normal. Neither tubercle bacilli, meningococci, or other pyogenic micrococci are demonstrated, and only a very small number of Gram-negative bacilli are seen. The absence of pus, or even an excess of leucocytes, together with a reduction of Fehling's solution, might suggest that the case is not an infection with meningococci or other pyogenic cocci, but it may possibly be of the tuberculous type, the excess of albumen is the only pathogenic featu

Hospital Case II.

L. W., aged 16, was quite well on Sunday, September 10th. When he got up on Monday he had pains in the back and neck, but there was no vomiting. He went to work, but his legs felt but there was no vomiting. He went to
weak. He shivered when he got home.

On September 11th the pains were worse in the back and waist, and he had marked girdle pain. He was constipated, and temperature was somewhat raised. He could not bear to have his head raised on account of the pain.

On September 11th he found he was losing power in his legs, and by September 12th he could not stand. He was admitted to hospital on September 16th, when Mr. Smith made the following notes :

"Paresis of legs-unable to move right leg-knee-jerk absent. Babinski present. No ankle clonus-unable to draw the leg up-slight power of extension. Left leg: Knee-jerk absentBabinski present. More power in this leg than the other. Can both flex and extend the knee though the movements are weak. Arms: No loss of power. No impairment of sensation. No Arms: No loss of power. No impairment of sensation. No involvement of bladder or rectum. Abdominal and cremasteric reflexes absent. Pupils equal, react to light. Tache
cérébrale. Pains along the spine and around waist. Temperacérébrale. Pains along the spine and around waist. Temperato give injections of heroin.

Report on the Spinal Fluid.-_"The fluid is faintly alkaline and fairly albuminous; it gives a practically normal reduction with Fehling's solution. The centrifugalized deposit is small in amount and shows a small number of blood discs, a few endothelial cells, and some leucocytes. The latter are distinctly in excess, but not in sufficient numbers to constitute pus. A mononuclear leucocytes. Smear preparations show an occasional Gram-positive micrococcus and a few Gram-negative bacillary organisms. Neither meningococci nor tubercle bacilli are found, but the characters of the fluid are suggestive of the tuberculous type of the disease."

Later Report. - The staphylococcus isolated from this specimen has the cultural characters of the Staphylococcus epidermidi. albus. The bacillus is of saprophytic type. The coccus, also, is probably derived from the skin, or some other extraneous source."

\section{Hospital Case iri.}

J. J., aged 7, sent me by Dr. Hodgson, of Alresford, with the following notes:

"Taken ill on September 17th. First seen on 18th. Com plained of stiffness overnight in legs and back. Could not stand. Temperature slightly raised. There was a history of a feverish attack about a month previously. Loss of power of the extensor muscles of the right leg and thigh, with partial the extensor muscles of the right leg and thigh, with partial the series of anaesthesia, though there was often hyperaesthesia, the series of anaesthesia, though there was often hyperaesthesia,
both general and localized, in the affected limbs.) Knee-jerks both general and localized, in the affected limbs.) Knee-jerks increased on right side-absent on left. Extensor response on
right side (Babinski). September 24th : Acute pain in right leg. right side (Babinski). September 24 th : Acute pain in right leg.
Marked retraction amounting to opisthotonos, the boy lying on Marked retraction amounting to opisthotonos, the boy lying on
his side $w$ : th the legs drawn up and head retracted; great pain 
being caused on raising the head, or on attempting to straighten the legs."

On admission (October 15th), the retraction and the pains had subsided, but on attempting any passive flexion of the legs the boy at once twisted over to the opposite side, sharply arching his back, though he could bear them fully extended if lying on his back. Any attempt to obtain Kernig's sign produced this curious contraction of the spinal muscles, and was evidently very painful.

The state of the reflexes was as Dr. Hodgson had already noted,

There was much loss of power in the extensor muscles of the thigh on the right side, and as in all cases in which the patellar reflexes were lost there was an extraordinary laxity of the patella tendon, so that the fingers could be inserted between that bone and the head of the tibia.

I have not noticed this phenomenon in so marked a degree in ordinary cases of loss of knee-jerk, and I take it that it may be looked upon as an index of the degree to which the extensor muscles have suffered.

\section{REMARKS.}

Here, then, we have what may be considered as a fairly widespread epidemic of acute disorder affecting the spinal cord and meninges, and although we have heard a good deal of late of epidemic acute anterior poliomyelitis, I think that these cases do not all conform to the type which we have been accustomed to meet with during the last twenty years, inasmuch as the intense pain in the back of the head and neck, the twitchings of the face, the retraction of the head, amounting in some cases to opisthotonos, the marked rigidity, and continued pains in the limbs, the interference with the due control over the bladder and rectum, and, in several instances, the more or less rapid and com. plete recovery, are quite contrary to the usual experience in that disease.

I had the advantage of meeting Sir James Goodhart in consultation over A. G., my fifth case, and he was decidedly of the opinion that we had to deal with cerebro-spinal meningitis, which was the conclusion I had previously come to with regard to the other cases $I$ had then seen.

Sir William Osler makes the statement that those instances of acute basic meningitis, with which from time to time we have met in such a severe and fulminating form, are nothing inore or less than sporadic cases of cerebro-spinal meningitis.

There are doubtless several signs which have been looked upon as more or less classical of this disease which were absent in my cases, notably the rash from which the old name of "spotted fever" was derived, but even in the severe basic form, of which I have seen a few instances, I cannot call to mind any rash. Where this occurs it indicates a malignant form of the disease, and a very severe toxaemia in addition to the local signs. Vomiting also in my series was unusual, although it did occur in Case v.

It may be that when, as in the epidemic form, the disease becomes generalized, it at the same time loses some of its virulence (and abortive cases do occur) but it is interesting to note that the older the victims the more severe and lasting has been the mischief-one of my cases (Case I, seen with Mr. Way) having died on about the fourth day, while the two youngest (Cases III and v), have apparently recovered without any trace of the paralysis occurring after acute anterior poliomyelitis.

So far I have not come across any cases in adult females, nor have I observed any connexion between this epidemic and the incidence of herpes zoster referred to by Dr. Garrow of Maryport in Cumbirland, in an epidemic of poliomyelitis which occurred there in August and Septem. ber, 1910. This was also noticed in the Cornwall epidemic this year.

I do not wish to give the impression that every case in the present epidemic is cerebro-spinal fever, but at least I wculd submit that anterior poliomyelitis is a misnomer, since the meningeal symptoms, either cerebral or spinal, have been most marked. Cases I, IV, and v in the private series, together with Nos. I and II of the hospital cases, present clinically the picture of cerebrospinal fever.

With regard to the pathology of cerebro-spinal fever there are three cocci which have been claimed by their discoverers as being the specific cause: (1) Weichselbaum's Diplococcus intracellularis; (2) Still's meningococcus (this in sporadic cases); (3) a micro-organism resembling the pneumococcus of croupous pneumonia; and I am fully aware of the absence of any pathological evidence in $\mathrm{my}$ cases as to the presence or otherwise of any of these organisms. Houston and Rankin's recent researches go to prove that the two former are quite distinct.

In Dr. Tullis's cases the specific oryanisms were not found, though albumen was present in the spinal fluid, which probably indicated inflammation of the meninges, notwithstanding that the normal reducing action of the fluid on cupric oxide was present, which reaction in cases of meningeal inflammation is generally absent. Had the cases come under observation during the very early stage, something more might have been learnt.

It would be interesting to know in what percentage of cases during the various epidemics reported it has been possible to examine the spinal fluid in the acute and early stage, since bacteriologists I presume would not admit the existence of cerebro-spinal meningitis without the presence of one of the above-mentioned organisms; but of this I am convinced, that, notwithstanding Dr. Allen Starr's classical description in Allbutt's System of acute anterior poliomyelitis, which I must admit describes very nearly some of the milder cases I have seen, we have here at any rate in some of the cases a distinct clinical entity, which is much more serious to life.

Pain! pain! has been the persistent outcry in all the cases, sometimes persisting for weeks, and as that acute observer, the late Dr. Bristowe, remarks of anterior poliomyelitis that " pain is no essential feature of its clinical history," and that "an almost total absence of pain may be regarded as characteristic of this disease," it is, I think, fairly evident that we have in this present epidemic a condition of things which, to quote from Dr. Ormerod, we may at least say, "We have had (until now) very scanty experience."

Now, while I fully concede that bacteriology and modern laboratory research bear a very great and increasing value in determining the hitherto obscure origin of many diseases, as an old practitioner I very much deprecate the fact that clinical observation is often made to take a back seat to the newer methods of diagnosis, and although, as I said before, several of my cases approximate more nearly the type of acute anterior poliomyelitis, there are in some (notably I, IV, and v) symptoms which correspond almost exactly to the "simple type" of cerebro-spinal fever which is described in Allbutt's System of Medicine.

\section{EPIDEMIC ANTERIOR POLIOMYELITIS IN SOUTH DERBYSHIRE.}

BY JOHN HAY MOIR, M.D., D.P.H., NEWHALL, BURTON-ON-TRENT.

IN the general interest evolved by the recent epidemic of anterior poliomyelitis during the past summer, a short synopsis of 25 cases which occurred in this district in South Derbyshire may be of some value.

All the cases occurred within the Urban District of Swadlincote, which comprises a population of 22,000 , scattered over an area of about three square miles. The principal industries are coal mining and earthenware manufacture. During the summer work is bad, and many families are in a state bordering on destitution. Dr. R. A Parkhill has kindly supplied me with the records of 14 cases which came under his care, the remainder being under my own observation.

There were two periods of greatest incidence, the first early in June amongst Dr. Parkhill's patients in the southwest of the district, the second early in August in the north-west. The epidemic apparently spread in this direction through the district, but there is no evidence to show the mode of progress. The distribution of cases was very scattered, with some exceptions, namely, 3 cases occurred in one street, and 2 in each of two houses. There was practically no communication between the houses affected, except possibly where the three cases occurred in one street. Twelve cases occurred in very poor and dirty houses. Dusty roads do not appear to be of any etiological moment. Many of the patients were strong and well nourished. The occurrence in both sexes was of 